\title{
BIHARMONIC GREEN FUNCTION OF A RING DOMAIN
}

\author{
TATYANA S. VAITEKHOVICH*
}

\begin{abstract}
A biharmonic Green function of a circular ring domain $R=\{z \in \mathrm{C}: 0<r<|z|<1\}$ is found in the form

$$
\widehat{G}_{2}(z, \zeta)=|\zeta-z|^{2} G_{1}(z, \zeta)+\widehat{h}_{2}(z, \zeta),
$$

where $G_{1}(z, \zeta)$ is the harmonic Green function of the ring $R$, and $\widehat{h}_{2}(z, \zeta)$ is a specially constructed biharmonic function.
\end{abstract}

\section{Introduction}

Green functions for differential operators are an important tool as well for the existence theory of boundary value problems for partial differential equations, as for the representation of solutions of such problems (see [3], [4], [5], [6], [9], [12], [16]). Among the operators for which it is interesting to define Green functions is the biharmonic operator

$$
\left(\partial_{z} \partial_{\bar{z}}\right)^{2}
$$

It is a fourth-order differential operator naturally arising in different problems of mechanics, for instance, in the linear elasticity theory, see e.g. [15].

There are two main approaches to determine a Green function for the biharmonic operator. The first one is to define the biharmonic Green function as the convolution of the harmonic Green function with itself, see e.g. [3], [6]. This leads to an equivalent definition of the biharmonic Green function as a solution to a certain Dirichlet problem for the Poisson equation.

The second approach is connected with the name of Almansi, who introduced at the end of 19th century [2] (see also [3], [6]) a concept of polyharmonic Green functions for the unit disk on the complex plane. The Almansi biharmonic Green function is different from the one mentioned above in its boundary behavior.

\footnotetext{
* The author is grateful for the support through a scholarship from the "Abgeordnetenhaus von Berlin" and expresses herewith her sincere thanks to Professor Heinrich Begehr.

Received 26 August 2008.
} 
The complex analytic approach gives the possibility to construct Green functions in explicit form for special domains. It is used to obtain the biharmonic Green functions in the case of the unit disk in [3].

In this paper a biharmonic Green function defined by convolution of the harmonic Green function with itself is constructed for a circular ring domain

$$
R:=\{z \in \mathrm{C}: 0<r<|z|<1\} .
$$

It has appeared that the method of direct evaluation of the biharmonic Green function used in [3] for the unit disk is not effective in the case of the circular ring as it leads to complicated calculations. From the definition the representation of the biharmonic Green function in the form

$$
\widehat{G}_{2}(z, \zeta)=|\zeta-z|^{2} G_{1}(z, \zeta)+\widehat{h}_{2}(z, \zeta),
$$

follows, where $G_{1}(z, \zeta)$ is the harmonic Green function for the ring $R$ and $\widehat{h}_{2}(z, \zeta)$ is a certain biharmonic function, which has to be defined.

The obtained biharmonic Green function is used to represent the solution of a related Dirichlet problem to the biharmonic equation. It should be noted that the biharmonic Green function for the ring domain $R$ given here is expressed by infinite series. It differs from the Green function of the circular ring domain for the bi-Laplace operator constructed in [11] with particular Dirichlet boundary condition in terms of certain new transcendental functions, which generalize the Weierstrass $\zeta$-function.

\section{Preliminaries and Notations}

Complex partial differential operators of the first order are defined by

$$
\partial_{z}=\frac{1}{2}\left(\partial_{x}-i \partial_{y}\right), \quad \partial_{\bar{z}}=\frac{1}{2}\left(\partial_{x}+i \partial_{y}\right), \quad z=x+i y, \quad x, y \in \mathrm{R},
$$

where $\bar{z}=x-i y$. The differential operator of the second order $4 \partial_{z} \partial_{\bar{z}}$ is called the complex Laplace operator.

A complex-valued function $w$, satisfying the differential equation

$$
\partial_{z} \partial_{\bar{z}} w=0
$$

in a open domain $D \subset \mathrm{C}$ is called a harmonic function in $D$.

In the same way, the operator $16\left(\partial_{z} \partial_{\bar{z}}\right)^{2}$ is refered to as the complex biLaplace or the biharmonic operator, and a complex-valued function $w$, for which

$$
\left(\partial_{z} \partial_{\bar{z}}\right)^{2} w=0
$$

holds in a open domain $D \subset \mathrm{C}$ is called a biharmonic function in $D$. 
A domain $D$ on the complex plane $\mathrm{C}$ is called a regular domain if it is bounded with a (piecewise) smooth boundary $\partial D$.

Let us formulate one of the fundamental theorems in the theory of complex partial differential equations, see e.g. [4], [5], [17].

Theorem 2.1 (Gauss Theorem: complex form). Let $D \subset \mathrm{C}$ be a regular domain, $w \in C^{1}(D ; \mathrm{C}) \cap C(\bar{D} ; \mathrm{C})$, then

$$
\int_{D} \partial_{\bar{z}} w(z) d x d y=\frac{1}{2 i} \int_{\partial D} w(z) d z
$$

and

$$
\int_{D} \partial_{z} w(z) d x d y=-\frac{1}{2 i} \int_{\partial D} w(z) d \bar{z}
$$

Definition 2.2. A real-valued function $G(z, \zeta)=\frac{1}{2} G_{1}(z, \zeta)$ in a regular domain $D \subset \subset$ is called the harmonic Green function of $D$, or more exactly the Green function of $D$ for the Laplace operator, if it possesses for any fixed $\zeta \in D$ as a function of $z$ the following properties:

$1^{\circ} G(z, \zeta)$ is harmonic in $D \backslash\{\zeta\}$,

$2^{\circ} G(z, \zeta)+\log |\zeta-z|$ is harmonic in $D$,

$3^{\circ} \lim _{z \rightarrow \partial D} G(z, \zeta)=0$.

The harmonic Green function has the additional properties, see e.g. [5]:

$4^{\circ} G(z, \zeta)>0$,

$5^{\circ} G(z, \zeta)=G(\zeta, z)$.

$6^{\circ}$ It is uniquely determined by $1^{\circ}-3^{\circ}$.

Not any domain on the complex plane has a harmonic Green function. The existence of the Green function for a given domain $D \subset \mathrm{C}$ can be proved in the case when the Dirichlet problems for harmonic function is solvable in $D$ (see, e.g. [5]). In the case of a regular simply connected domain the harmonic Green function can be found from the well-known Green function of the unit disk by using the corresponding conformal mapping, see e.g. [5]. In the case of multiply connected domains the situation is much more complicated, see e.g. [1], [10], [15]-[17].

If a domain $D$ on the complex plane possesses the harmonic Green function $G_{1}(z, \zeta)$, then any function $w \in C^{2}(D ; \mathrm{C}) \cap C^{1}(\bar{D} ; \mathrm{C})$ can be represented in the following form (see, e.g. [14]) 
(2) $w(z)=-\frac{1}{4 \pi} \int_{\partial D} w(\zeta) \partial_{\nu_{\zeta}} G_{1}(z, \zeta) d s_{\zeta}$

$$
-\frac{1}{\pi} \int_{D} \partial_{\zeta} \partial_{\bar{\zeta}} w(\zeta) G_{1}(z, \zeta) d \xi d \eta, \quad \zeta=\xi+i \eta,
$$

where $\partial_{\nu_{\zeta}}$ denotes the outward normal derivative and $s_{\zeta}$ is the arc length parameter on $\partial D$ with respect to the variable $\zeta$.

The harmonic Green function is a fundamental solution for the Laplace operator. Together with the representation formula (2) it serves to solve the Dirichlet problem for the Poisson equation, i.e.

$$
\partial_{z} \partial_{\bar{z}} w=f \text { in } D, \quad w=\gamma \text { on } \partial D,
$$

where $f \in L_{2}(D ; \mathrm{C}) \cap C(D ; \mathrm{C}), \gamma \in C(\partial D ; \mathrm{C})$ are given, see e.g. [13]. The solution of the problem (3) is unique and expressed by

(4) $w(z)=-\frac{1}{4 \pi} \int_{\partial D} \partial_{\nu_{\zeta}} G_{1}(z, \zeta) \gamma(\zeta) d s_{\zeta}-\frac{1}{\pi} \int_{D} G_{1}(z, \zeta) f(\zeta) d \xi d \eta$.

Let us insert the Green function $G_{1}(\cdot, \zeta)$ in (4) instead of $f$ and denote

$$
\widehat{G}_{2}(z, \zeta):=-\frac{1}{\pi} \int_{D} G_{1}(z, \widetilde{\zeta}) G_{1}(\widetilde{\zeta}, \zeta) d \widetilde{\xi} d \widetilde{\eta} .
$$

Comparing this with formula (4), one observes that the introduced function $\widehat{G}_{2}(\cdot, \zeta)$ is the solution to the Dirichlet problem

$$
\partial_{z} \partial_{\bar{z}} \widehat{G}_{2}(z, \zeta)=G_{1}(z, \zeta) \text { in } D, \quad \widehat{G}_{2}(z, \zeta)=0 \text { on } \partial D,
$$

for any $\zeta \in D$.

Definition 2.3. A real-valued function $\widehat{G}_{2}(z, \zeta)$ in a regular domain $D \subset$ $C$ is called a biharmonic Green function of $D$, or more exactly a Green function of $D$ for the bi-Laplace operator, if it satisfies for any fixed $\zeta \in D$ as a function of $z$ the following properties:

$1^{\circ} \widehat{G}_{2}(z, \zeta)$ is biharmonic in $D \backslash\{\zeta\}$,

$2^{\circ} \widehat{G}_{2}(z, \zeta)+|\zeta-z|^{2} \log |\zeta-z|^{2}$ is biharmonic in $D$,

$3^{\circ} \widehat{G}_{2}(z, \zeta)=0, \partial_{z} \partial_{\bar{z}} \widehat{G}_{2}(z, \zeta)=0$ for $z \in \partial D$.

The function $\widehat{G}_{2}$ defined in (5) represents such a biharmonic Green function of $D$, what follows from the properties of the harmonic Green function of $D$.

From the properties $1^{\circ}-3^{\circ}$ the symmetry of a biharmonic Green function can be derived, i.e.

$$
4^{\circ} \widehat{G}_{2}(z, \zeta)=\widehat{G}_{2}(\zeta, z) \text { for } z \neq \zeta
$$


Contrary to $\widehat{G}_{2}(z, \zeta)$ the mentioned biharmonic Green-Almansi function $G_{2}(z, \zeta)$ (see, e.g. [2], [3], [7], [8], [11]) is not a primitive of $G_{1}(z, \zeta)$ with respect to the Laplace operator. It satisfies the properties $1^{\circ}, 2^{\circ}, 4^{\circ}$, but has different boundary behavior, namely

$$
G_{2}(z, \zeta)=0, \partial_{\nu_{z}} G_{2}(z, \zeta)=0 \quad \text { for } \quad z \in \partial D, \zeta \in D .
$$

In the case of the unit disk the explicit form for $\widehat{G}_{2}(z, \zeta)$ is given in [3], [6], [9], for $G_{2}(z, \zeta)$ it is presented in [2] (see also [3], [6], [9]). For a circular ring $G_{2}(z, \zeta)$ is obtained in [11].

Our aim is to obtain the biharmonic Green function $\widehat{G}_{2}(z, \zeta)$ for a circular ring domain $R=\{z \in \mathrm{C}: 0<r<|z|<1\}$. For this domain the harmonic Green function exists and is expressed by $G(z, \zeta)=\frac{1}{2} G_{1}(z, \zeta)$ with

(7) $G_{1}(z, \zeta)=\frac{\log |z|^{2} \log |\zeta|^{2}}{\log r^{2}}-\log \left|\frac{\zeta-z}{1-z \bar{\zeta}} \prod_{k=1}^{\infty} \frac{\left(z-r^{2 k} \zeta\right)\left(\zeta-r^{2 k} z\right)}{\left(z \bar{\zeta}-r^{2 k}\right)\left(1-r^{2 k} z \bar{\zeta}\right)}\right|^{2}$,

see [1], [10], [16], [17].

It has appeared that the derivation of the exact expression for the biharmonic Green function of the circular ring domain $R$ from the definition (formula (5)) is extremely involved because of computational complexity. Therefore another method is required. Our approach is based on the following idea. The biharmonic Green function $\widehat{G}_{2}(z, \zeta)$ in $D \backslash\{\zeta\}$ can be represented in the form

$$
\widehat{G}_{2}(z, \zeta)=|\zeta-z|^{2} G_{1}(z, \zeta)+\widehat{h}_{2}(z, \zeta),
$$

with $\widehat{h}_{2}(z, \zeta)$ being a biharmonic function in $D$. This representation follows from the second property of $\widehat{G}_{2}(z, \zeta)$, the second property of $G(z, \zeta)$, according to which

$$
2 G(z, \zeta)=G_{1}(z, \zeta)=-\log |\zeta-z|^{2}+h_{1}(z, \zeta)
$$

with $h_{1}(z, \zeta)$ being a harmonic function in $D$. Thus to get the expression for $\widehat{G}_{2}(z, \zeta)$ the function $\widehat{h}_{2}(z, \zeta)$ has to be found.

As $\widehat{G}_{2}(z, \zeta)$ is the solution to the Dirichlet problem (6), it follows that the function $\widehat{h}_{2}(z, \zeta)$ is the solution to the Dirichlet problem

(9) $\partial_{z} \partial_{\bar{z}} \widehat{h}_{2}(z, \zeta)=2 \operatorname{Re}\left[(\zeta-z) \partial_{z} G_{1}(z, \zeta)\right]$ in $D, \quad \widehat{h}_{2}(z, \zeta)=0$ on $\partial D$,

for any $\zeta \in D$. To find the solution of this problem formula (4) is used. According to it

$$
\widehat{h}_{2}(z, \zeta)=-\frac{2}{\pi} \int_{D} \operatorname{Re}\left[(\zeta-\widetilde{\zeta}) \partial_{\widetilde{\zeta}} G_{1}(\widetilde{\zeta}, \zeta)\right] G_{1}(z, \widetilde{\zeta}) d \widetilde{\xi} d \widetilde{\eta},
$$


or equivalently

$$
\widehat{h}_{2}(z, \zeta)=2 \operatorname{Re}\left[\frac{1}{\pi} \int_{D}(\widetilde{\zeta}-\zeta) \partial_{\widetilde{\zeta}} G_{1}(\widetilde{\zeta}, \zeta) G_{1}(z, \widetilde{\zeta}) d \widetilde{\xi} d \widetilde{\eta}\right],
$$

as the harmonic Green function $G_{1}(z, \zeta)$ is real-valued.

\section{Construction of the biharmonic Green function of a circular ring domain}

In order to obtain the biharmonic Green function of a circular ring domain $R=\{z \in \mathrm{C}: 0<r<|z|<1\}$, the corresponding function $\widehat{h}_{2}(z, \zeta)$ defined in (10) has to be found. By using the explicit form of the harmonic Green function of $R$ given in (7) the formula (10) can be rewritten as

$$
\begin{gathered}
\widehat{h}_{2}(z, \zeta)=2 \operatorname{Re}\left[\frac { 1 } { \pi } \int _ { R } ( \widetilde { \zeta } - \zeta ) \left[\frac{\log |\zeta|^{2}}{\log |r|^{2}} \frac{1}{\widetilde{\zeta}}-\frac{1}{\widetilde{\zeta}-\zeta}+\frac{\bar{\zeta}}{\widetilde{\zeta} \bar{\zeta}-1}\right.\right. \\
\left.\left.+\sum_{n=1}^{\infty}\left(\frac{r^{2 n} \bar{\zeta}}{r^{2 n} \widetilde{\zeta} \bar{\zeta}-1}+\frac{\bar{\zeta}}{\widetilde{\zeta} \bar{\zeta}-r^{2 n}}-\frac{1}{\widetilde{\zeta}-r^{2 n} \zeta}-\frac{r^{2 n}}{r^{2 n} \widetilde{\zeta}-\zeta}\right)\right] G_{1}(z, \widetilde{\zeta}) d \widetilde{\xi} d \widetilde{\eta}\right] \\
(11) \quad=2 \operatorname{Re}\left[\frac { 1 } { \pi } \int _ { R } \left[\frac{\log |\zeta|^{2}}{\log |r|^{2}} \frac{\widetilde{\zeta}-\zeta}{\widetilde{\zeta}}+\frac{1-|\zeta|^{2}}{\widetilde{\zeta} \bar{\zeta}-1}+\sum_{n=1}^{\infty}\left(\frac{1-r^{2 n}|\zeta|^{2}}{r^{2 n} \widetilde{\zeta} \bar{\zeta}-1}\right.\right.\right. \\
\left.\left.\left.-\frac{r^{2 n}-|\zeta|^{2}}{r^{2 n}-\widetilde{\zeta} \bar{\zeta}}+\frac{\left(1-r^{2 n}\right) \zeta}{\widetilde{\zeta}-r^{2 n} \zeta}-\frac{\left(1-r^{2 n}\right) \zeta}{r^{2 n} \widetilde{\zeta}-\zeta}\right)\right] G_{1}(z, \tilde{\zeta}) d \widetilde{\xi} d \widetilde{\eta}\right] .
\end{gathered}
$$

The evaluation of the integral (11) is proceeded in five steps. The calculations are represented here just briefly. The main facts which are used for getting the final results of the evaluations are Gauss theorem, Cauchy theorem and the formula

$w(z)=-\frac{1}{4 \pi i} \int_{\partial R}|\zeta|^{2} \partial_{\nu_{\zeta}} G_{1}(z, \zeta) w(\zeta) \frac{d \zeta}{\zeta}-\frac{1}{\pi} \int_{R} G_{1}(z, \zeta) \partial_{\zeta} \partial_{\zeta} w(\zeta) d \xi d \eta$,

which is (2) for $D=R$.

1. Let us consider the integral

$$
\begin{aligned}
I_{1}(z, \zeta) & :=2 \operatorname{Re}\left[\frac{1}{\pi} \int_{R} \frac{\widetilde{\zeta}-\zeta}{\widetilde{\zeta}} G_{1}(z, \tilde{\zeta}) d \tilde{\xi} d \tilde{\eta}\right] \\
& =\frac{1}{\pi} \int_{R}\left[\frac{\widetilde{\zeta}-\zeta}{\widetilde{\zeta}}+\frac{\widetilde{\zeta}-\zeta}{\widetilde{\zeta}}\right] G_{1}(z, \tilde{\zeta}) d \tilde{\xi} d \tilde{\eta},
\end{aligned}
$$


which is related to the first part in (11).

Since

$$
\partial_{z} \partial_{\bar{z}}\left[2|z|^{2}-(z \bar{\zeta}+\bar{z} \zeta) \log |z|^{2}\right]=\frac{z-\zeta}{z}+\frac{\overline{z-\zeta}}{\bar{z}},
$$

by formula (12) we get

$$
I_{1}(z, \zeta)=(z \bar{\zeta}+\bar{z} \zeta) \log |z|^{2}-2|z|^{2}-\widehat{I}_{1}(z, \zeta)
$$

where

$$
\begin{gathered}
\widehat{I}_{1}(z, \zeta):=\frac{1}{4 \pi i} \int_{\partial R}\left[2|\tilde{\zeta}|^{2}-(\widetilde{\zeta} \bar{\zeta}+\widetilde{\zeta} \zeta) \log |\widetilde{\zeta}|^{2}\right]|\widetilde{\zeta}| \partial_{\nu_{\tilde{\zeta}}} G_{1}(z, \widetilde{\zeta}) \frac{d \widetilde{\zeta}}{\widetilde{\zeta}} \\
=\operatorname{Re}\left[\frac { 1 } { 2 \pi i } \int _ { \partial R } [ 2 | \widetilde { \zeta } | ^ { 2 } - ( \widetilde { \zeta } \overline { \zeta } + \widetilde { \widetilde { \zeta } \zeta } ) \operatorname { l o g } | \widetilde { \zeta } | ^ { 2 } ] \left[\frac{\log |z|^{2}}{\log r^{2}} \frac{1}{\widetilde{\zeta}}-\frac{1}{\widetilde{\zeta}-z}-\frac{\bar{z}}{1-\bar{z} \widetilde{\zeta}}\right.\right. \\
\left.\left.+\sum_{k=1}^{\infty}\left(\frac{r^{2 k} \bar{z}}{r^{2 k} \bar{z} \widetilde{\zeta}-1}+\frac{\bar{z}}{\bar{z} \widetilde{\zeta}-r^{2 k}}-\frac{1}{\widetilde{\zeta}-r^{2 k} z}-\frac{r^{2 k}}{r^{2 k} \widetilde{\zeta}-z}\right)\right] d \widetilde{\zeta}\right] \\
=2 \operatorname{Re}\left[\left(1-r^{2}\right) \frac{\log |z|^{2}}{\log r^{2}}+\frac{r^{2}}{1-r^{2}} \log r^{2}\left(\frac{\bar{\zeta}}{\bar{z}}-z \bar{\zeta}\right)\right] .
\end{gathered}
$$

Then the first part in (11) is

$$
\begin{gathered}
2 \operatorname{Re}\left[\frac{\log |\zeta|^{2}}{\log r^{2}} \frac{1}{\pi} \int_{R} \frac{\widetilde{\zeta}-\zeta}{\widetilde{\zeta}} G_{1}(z, \tilde{\zeta}) d \tilde{\xi} d \tilde{\eta}\right] \\
=\frac{\log |\zeta|^{2}}{\log r^{2}} I_{1}(z, \zeta)=2 \operatorname{Re}\left[\frac { \operatorname { l o g } | \zeta | ^ { 2 } } { \operatorname { l o g } r ^ { 2 } } \left(1-|z|^{2}+z \bar{\zeta} \log |z|^{2}\right.\right. \\
\left.\left.-\left(1-r^{2}\right) \frac{\log |z|^{2}}{\log r^{2}}-\frac{r^{2}}{1-r^{2}} \log r^{2}\left(\frac{\bar{\zeta}}{\bar{z}}-z \bar{\zeta}\right)\right)\right] .
\end{gathered}
$$

2. In the next step we evaluate the integral

$$
\begin{aligned}
I_{2}(z, \zeta) & :=2 \operatorname{Re}\left[\frac{1}{\pi} \int_{R} \frac{1}{r^{2 n} \widetilde{\zeta} \bar{\zeta}-1} G_{1}(z, \widetilde{\zeta}) d \widetilde{\xi} d \widetilde{\eta}\right] \\
& =\frac{1}{\pi} \int_{R}\left[\frac{1}{r^{2 n} \widetilde{\zeta} \bar{\zeta}-1}+\frac{1}{r^{2 n} \bar{\zeta} \zeta-1}\right] G_{1}(z, \widetilde{\zeta}) d \widetilde{\xi} d \tilde{\eta}
\end{aligned}
$$

for any fixed $n \in \mathrm{N}$. 
Observing that

$$
\partial_{z} \partial_{\bar{z}}\left[\frac{1}{r^{2 n}}\left(\frac{z}{\zeta}+\frac{\bar{z}}{\bar{\zeta}}\right) \log \left|1-r^{2 n} z \bar{\zeta}\right|^{2}\right]=\frac{1}{r^{2 n} z \bar{\zeta}-1}+\frac{1}{r^{2 n} \bar{z} \zeta-1},
$$

by formula (12) one gets

$$
I_{2}(z, \zeta)=-\frac{1}{r^{2 n}}\left(\frac{z}{\zeta}+\frac{\bar{z}}{\bar{\zeta}}\right) \log \left|1-r^{2 n} z \bar{\zeta}\right|^{2}-\frac{1}{r^{2 n}} \widehat{I}_{2}(z, \zeta),
$$

with

$$
\begin{gathered}
\widehat{I}_{2}(z, \zeta):=\frac{1}{4 \pi i} \int_{\partial R}\left(\frac{\widetilde{\zeta}}{\zeta}+\frac{\bar{\zeta}}{\bar{\zeta}}\right) \log \left|1-r^{2 n} \widetilde{\zeta} \bar{\zeta}\right|^{2}|\widetilde{\zeta}| \partial_{\mho_{\zeta}} G_{1}(z, \widetilde{\zeta}) \frac{d \widetilde{\zeta}}{\widetilde{\zeta}} \\
=\operatorname{Re}\left[\frac { 1 } { 2 \pi i } \int _ { \partial R } \widetilde { \zeta } ( \frac { \widetilde { \zeta } } { \zeta } + \frac { \widetilde { \zeta } } { \overline { \zeta } } ) \left[\frac{\log |z|^{2}}{\log r^{2}} \frac{1}{\widetilde{\zeta}}-\frac{1}{\widetilde{\zeta}-z}-\frac{\bar{z}}{1-\bar{z} \widetilde{\zeta}}\right.\right. \\
\left.\left.+\sum_{k=1}^{\infty}\left(\frac{r^{2 k} \bar{z}}{r^{2 k} \bar{z} \widetilde{\zeta}-1}+\frac{\bar{z}}{\bar{z} \widetilde{\zeta}-r^{2 k}}-\frac{1}{\widetilde{\zeta}-r^{2 k} z}-\frac{r^{2 k}}{r^{2 k} \widetilde{\zeta}-z}\right)\right] \log \left|1-r^{2 n} \widetilde{\zeta} \bar{\zeta}\right|^{2} \frac{d \widetilde{\zeta}}{\widetilde{\zeta}}\right] \\
=-2 \operatorname{Re}\left[\left(1-r^{2}\right) r^{2 n} \frac{\log |z|^{2}}{\log r^{2}}+\left(\frac{\bar{z}}{\bar{\zeta}}+\frac{1}{z \bar{\zeta}}\right) \log \left(1-r^{2 n} z \bar{\zeta}\right)\right. \\
\left.-\left(1-r^{2}\right) \sum_{k=1}^{\infty}\left(\frac{z}{r^{2 k} \zeta} \log \left(1-\frac{r^{2(n+k)} \zeta}{z}\right)-\frac{1}{r^{2 k} z \bar{\zeta}} \log \left(1-r^{2(n+k)} z \bar{\zeta}\right)\right)\right] .
\end{gathered}
$$

Then

$$
\begin{aligned}
& 2 \operatorname{Re}\left[\left(1-r^{2 n}|\zeta|^{2}\right) \frac{1}{\pi} \int_{R} \frac{1}{r^{2 n} \widetilde{\zeta} \bar{\zeta}-1} G_{1}(z, \widetilde{\zeta}) d \widetilde{\xi} d \widetilde{\eta}\right]=\left(1-r^{2 n}|\zeta|^{2}\right) I_{2}(z, \zeta) \\
& (14) \quad=-\frac{2\left(1-r^{2 n}|\zeta|^{2}\right)}{r^{2 n}} \operatorname{Re}\left[\left(\frac{\bar{z}}{\bar{\zeta}}-\frac{1}{z \bar{\zeta}}\right) \log \left(1-r^{2 n} z \bar{\zeta}\right)-\left(1-r^{2}\right) r^{2 n} \frac{\log |z|^{2}}{\log |r|^{2}}\right. \\
& \left.+\left(1-r^{2}\right) \sum_{k=1}^{\infty}\left(\frac{z}{r^{2 k} \zeta} \log \left(1-\frac{r^{2(n+k)} \zeta}{z}\right)-\frac{1}{r^{2 k} z \bar{\zeta}} \log \left(1-r^{2(n+k)} z \bar{\zeta}\right)\right)\right] .
\end{aligned}
$$

3. The third integral is

$$
\begin{aligned}
I_{3}(z, \zeta): & =2 \operatorname{Re}\left[\frac{1}{\pi} \int_{R} \frac{1}{r^{2 n}-\widetilde{\zeta} \bar{\zeta}} G_{1}(z, \widetilde{\zeta}) d \tilde{\xi} d \widetilde{\eta}\right] \\
& =\frac{1}{\pi} \int_{R}\left[\frac{1}{r^{2 n}-\widetilde{\zeta} \bar{\zeta}}+\frac{1}{r^{2 n}-\widetilde{\zeta} \zeta}\right] G_{1}(z, \widetilde{\zeta}) d \widetilde{\xi} d \widetilde{\eta}
\end{aligned}
$$


where $n \in \mathrm{N}$ is fixed. As

$$
\partial_{z} \partial_{\bar{z}}\left[\left(\frac{z}{\zeta}+\frac{\bar{z}}{\bar{\zeta}}\right) \log \left|z \bar{\zeta}-r^{2 n}\right|^{2}\right]=-\left(\frac{1}{r^{2 n}-z \bar{\zeta}}+\frac{1}{r^{2 n}-\bar{z} \zeta}\right)
$$

by formula (12) it is

$$
I_{3}(z, \zeta)=\left(\frac{z}{\zeta}+\frac{\bar{z}}{\bar{\zeta}}\right) \log \left|z \bar{\zeta}-r^{2 n}\right|^{2}+\widehat{I_{3}}(z, \zeta)
$$

where

$$
\begin{aligned}
& \widehat{I_{3}}(z, \zeta):=\frac{1}{4 \pi i} \int_{\partial R}\left(\frac{\widetilde{\zeta}}{\zeta}+\frac{\widetilde{\zeta}}{\bar{\zeta}}\right) \log \left|\widetilde{\zeta} \bar{\zeta}-r^{2 n}\right|^{2}|\widetilde{\zeta}| \partial_{v_{\zeta}} G_{1}(z, \widetilde{\zeta}) \frac{d \widetilde{\zeta}}{\widetilde{\zeta}} \\
& =\operatorname{Re}\left[\frac { 1 } { 2 \pi i } \int _ { \partial R } \widetilde { \zeta } ( \frac { \widetilde { \zeta } } { \zeta } + \frac { \widetilde { \zeta } } { \overline { \zeta } } ) ( \operatorname { l o g } | | \widetilde { \zeta } | ^ { 2 } - \frac { r ^ { 2 n } \tilde { \zeta } } { \zeta } | ^ { 2 } + \operatorname { l o g } | \zeta | ^ { 2 } - \operatorname { l o g } | \widetilde { \zeta } | ^ { 2 } ) \left[\frac{\log |z|^{2}}{\log r^{2}} \frac{1}{\widetilde{\zeta}}\right.\right. \\
& \left.\left.-\frac{1}{\widetilde{\zeta}-z}-\frac{\bar{z}}{1-\bar{z} \widetilde{\zeta}}+\sum_{k=1}^{\infty}\left(\frac{r^{2 k} \bar{z}}{r^{2 k} \bar{z} \widetilde{\zeta}-1}+\frac{\bar{z}}{\bar{z} \widetilde{\zeta}-r^{2 k}}-\frac{1}{\widetilde{\zeta}-r^{2 k} z}-\frac{r^{2 k}}{r^{2 k} \widetilde{\zeta}-z}\right)\right] \frac{d \tilde{\zeta}}{\widetilde{\zeta}}\right] \\
& =-2 \operatorname{Re}\left[\left(1-r^{2}\right) \frac{z}{\zeta} \log \left(1-\frac{r^{2 n} z}{\zeta}\right)+\left(\frac{r^{2}}{z \bar{\zeta}}+\frac{\bar{z}}{\bar{\zeta}}\right) \log \left(1-\frac{r^{2 n}}{z \bar{\zeta}}\right)\right. \\
& +\frac{z}{\zeta} \log |\zeta|^{2}+\frac{r^{2}}{1-r^{2}}\left(\frac{1}{z \bar{\zeta}}-\frac{\bar{z}}{\bar{\zeta}}\right) \log r^{2} \\
& \left.-\left(1-r^{2}\right) \sum_{k=1}^{\infty}\left(\frac{r^{2 k}}{z \bar{\zeta}} \log \left(1-\frac{r^{2(n+k)}}{z \bar{\zeta}}\right)-\frac{r^{2 k} z}{\zeta} \log \left(1-\frac{r^{2(n+k)} z}{\zeta}\right)\right)\right] .
\end{aligned}
$$

Then

$$
\begin{aligned}
2 \operatorname{Re}\left[\left(r^{2 n}-|\zeta|^{2}\right) \frac{1}{\pi} \int_{R} \frac{1}{r^{2 n}-\tilde{\zeta} \bar{\zeta}} G_{1}(z, \widetilde{\zeta}) d \widetilde{\xi} d \widetilde{\eta}\right]=\left(r^{2 n}-|\zeta|^{2}\right) I_{3}(z, \zeta) \\
=2\left(r^{2 n}-|\zeta|^{2}\right) \operatorname{Re}\left[\left(\frac{\bar{z}}{\bar{\zeta}}-\frac{r^{2}}{z \bar{\zeta}}\right) \log \left(1-\frac{r^{2 n}}{z \bar{\zeta}}\right)-\left(1-r^{2}\right) \frac{z}{\zeta} \log \left(1-\frac{r^{2 n} z}{\zeta}\right)\right. \\
+\frac{z}{\zeta} \log |z|^{2}+\frac{r^{2}}{1-r^{2}}\left(\frac{\bar{z}}{\bar{\zeta}}-\frac{1}{z \bar{\zeta}}\right) \log r^{2} \\
\left.+\left(1-r^{2}\right) \sum_{k=1}^{\infty}\left(\frac{r^{2 k}}{z \bar{\zeta}} \log \left(1-\frac{r^{2(n+k)}}{z \bar{\zeta}}\right)-\frac{r^{2 k} z}{\zeta} \log \left(1-\frac{r^{2(n+k)} z}{\zeta}\right)\right)\right] .
\end{aligned}
$$


4. Now the integral

$$
\begin{aligned}
I_{4}(z, \zeta) & :=2 \operatorname{Re}\left[\frac{1}{\pi} \int_{R} \frac{1}{\widetilde{\zeta}-r^{2 n} \zeta} G_{1}(z, \widetilde{\zeta}) d \widetilde{\xi} d \widetilde{\eta}\right] \\
& =\frac{1}{\pi} \int_{R}\left[\frac{1}{\widetilde{\zeta}-r^{2 n} \zeta}+\frac{1}{\overline{\widetilde{\zeta}-r^{2 n} \zeta}}\right] G_{1}(z, \widetilde{\zeta}) d \tilde{\xi} d \widetilde{\eta},
\end{aligned}
$$

has to be found with $n \in \mathrm{N}$ fixed. Observing that

$$
\partial_{z} \partial_{\bar{z}}\left[(z+\bar{z}) \log \left|z-r^{2 n} \zeta\right|^{2}\right]=\frac{1}{z-r^{2 n} \zeta}+\frac{1}{\overline{z-r^{2 n} \zeta}},
$$

by formula (12) the equality

$$
I_{4}(z, \zeta)=-(z+\bar{z}) \log \left|z-r^{2 n} \zeta\right|^{2}-\widehat{I}_{4}(z, \zeta),
$$

is obtained, with

$$
\begin{gathered}
\widehat{I}_{4}(z, \zeta):=\frac{1}{4 \pi i} \int_{\partial R}(\widetilde{\zeta}+\widetilde{\zeta}) \log \left|\widetilde{\zeta}-r^{2 n} \zeta\right|^{2}|\widetilde{\zeta}| \partial_{v \widetilde{\zeta}} G_{1}(z, \widetilde{\zeta}) \frac{d \widetilde{\zeta}}{\widetilde{\zeta}} \\
=\operatorname{Re}\left[\frac{1}{2 \pi i} \int_{\partial R} \widetilde{\zeta} \widetilde{\zeta}+\widetilde{\zeta}\right)\left(\left.\log || \tilde{\zeta}\right|^{2}-\left.r^{2 n} \widetilde{\zeta} \bar{\zeta}\right|^{2}-\log |\widetilde{\zeta}|^{2}\right)\left[\frac{\log |z|^{2}}{\log r^{2}} \frac{1}{\widetilde{\zeta}}\right. \\
\left.\left.-\frac{1}{\widetilde{\zeta}-z}-\frac{\bar{z}}{1-\bar{z} \widetilde{\zeta}}+\sum_{k=1}^{\infty}\left(\frac{r^{2 k} \bar{z}}{r^{2 k} \bar{z} \widetilde{\zeta}-1}+\frac{\bar{z}}{\bar{z} \widetilde{\zeta}-r^{2 k}}-\frac{1}{\widetilde{\zeta}-r^{2 k} z}-\frac{r^{2 k}}{r^{2 k} \widetilde{\zeta}-z}\right)\right] \frac{d \widetilde{\zeta}}{\widetilde{\zeta}}\right] \\
=-2 \operatorname{Re}\left[\left(z+\frac{1}{\bar{z}}\right) \log \left(1-r^{2 n} z \bar{\zeta}\right)+\left(z+\frac{r^{2}}{\bar{z}}\right) \log \left(1-\frac{r^{2 n} \zeta}{z}\right)\right. \\
-\left(r^{2} z+\frac{1}{\bar{z}}\right) \log \left(1-r^{2 n} z \bar{\zeta}\right)-\frac{r^{2}}{1-r^{2}}\left(z-\frac{1}{\bar{z}}\right) \log r^{2} \\
\left.-\left(1-r^{2}\right) \sum_{k=1}^{\infty}\left(\frac{r^{2 k}}{z} \log \left(1-\frac{r^{2(n+k)} \zeta}{z}\right)-r^{2 k} z \log \left(1-r^{2(n+k)} z \bar{\zeta}\right)\right)\right] .
\end{gathered}
$$

Then

$$
\begin{aligned}
& 2 \operatorname{Re}\left[\left(1-r^{2 n}\right) \zeta \frac{1}{\pi} \int_{R} \frac{1}{\widetilde{\zeta}-r^{2 n} \zeta} G_{1}(z, \tilde{\zeta}) d \widetilde{\xi} d \widetilde{\eta}\right] \\
= & -2\left(1-r^{2 n}\right) \operatorname{Re}\left[z \bar{\zeta} \log |z|^{2}-\left(1-r^{2}\right) z \bar{\zeta} \log \left(1-r^{2 n} z \bar{\zeta}\right)\right.
\end{aligned}
$$




$$
\begin{gathered}
+\left(z \bar{\zeta}-\frac{r^{2} \bar{\zeta}}{\bar{z}}\right) \log \left(1-\frac{r^{2 n} \zeta}{z}\right)+\frac{r^{2}}{1-r^{2}}\left(z \bar{\zeta}-\frac{\bar{\zeta}}{\bar{z}}\right) \log r^{2} \\
\left.+\left(1-r^{2}\right) \sum_{k=1}^{\infty}\left(\frac{r^{2 k} \zeta}{z} \log \left(1-\frac{r^{2(n+k)} \zeta}{z}\right)-r^{2 k} z \bar{\zeta} \log \left(1-r^{2(n+k)} z \bar{\zeta}\right)\right)\right] .
\end{gathered}
$$

5. In the last step we evaluate

$$
\begin{aligned}
I_{5}(z, \zeta) & :=2 \operatorname{Re}\left[\frac{1}{\pi} \int_{R} \frac{1}{r^{2 n} \widetilde{\zeta}-\zeta} G_{1}(z, \widetilde{\zeta}) d \widetilde{\xi} d \widetilde{\eta}\right] \\
& =\frac{1}{\pi} \int_{R}\left[\frac{1}{r^{2 n} \widetilde{\zeta}-\zeta}+\frac{1}{r^{2 n} \widetilde{\zeta}-\zeta}\right] G_{1}(z, \widetilde{\zeta}) d \tilde{\xi} d \widetilde{\eta}
\end{aligned}
$$

with $n \in \mathrm{N}$ fixed. As

$$
\partial_{z} \partial_{\bar{z}}\left[\frac{1}{r^{2 n}}(z+\bar{z}) \log \left|r^{2 n} z-\zeta\right|^{2}\right]=\frac{1}{r^{2 n} z-\zeta}+\frac{1}{\overline{r^{2 n} z-\zeta}},
$$

using again the formula (12), one gets

$$
I_{5}(z, \zeta)=-\frac{1}{r^{2 n}}(z+\bar{z}) \log \left|r^{2 n} z-\zeta\right|^{2}-\frac{1}{r^{2 n}} \widehat{I}_{5}(z, \zeta),
$$

where

$$
\begin{gathered}
\widehat{I}_{5}(z, \zeta):=\frac{1}{4 \pi i} \int_{\partial R}(\widetilde{\zeta}+\overline{\widetilde{\zeta}}) \log \left|r^{2 n} \widetilde{\zeta}-\zeta\right|^{2}|\widetilde{\zeta}| \partial_{\nu_{\zeta}} G_{1}(z, \widetilde{\zeta}) \frac{d \widetilde{\zeta}}{\widetilde{\zeta}} \\
=\operatorname{Re}\left[\frac { 1 } { 2 \pi i } \int _ { \partial R } \widetilde { \zeta } ( \widetilde { \zeta } + \widetilde { \zeta } ) ( \operatorname { l o g } | 1 - \frac { r ^ { 2 n } \widetilde { \zeta } } { \zeta } | ^ { 2 } + \operatorname { l o g } | \zeta | ^ { 2 } ) \left[\frac{\log |z|^{2}}{\log r^{2}} \frac{1}{\widetilde{\zeta}}\right.\right. \\
\left.\left.-\frac{1}{\widetilde{\zeta}-z}-\frac{\bar{z}}{1-\bar{z} \widetilde{\zeta}}+\sum_{k=1}^{\infty}\left(\frac{r^{2 k} \bar{z}}{r^{2 k} \bar{z} \widetilde{\zeta}-1}+\frac{\bar{z}}{\bar{z} \widetilde{\zeta}-r^{2 k}}-\frac{1}{\widetilde{\zeta}-r^{2 k} z}-\frac{r^{2 k}}{r^{2 k} \widetilde{\zeta}-z}\right)\right] \frac{d \widetilde{\zeta}}{\widetilde{\zeta}}\right] \\
=-2 \operatorname{Re}\left[z \log |\zeta|^{2}+\left(z+\frac{1}{\bar{z}}\right) \log \left(1-\frac{r^{2 n} z}{\zeta}\right)+\left(1-r^{2}\right) \frac{r^{2 n}}{\zeta} \frac{\log |z|^{2}}{\log r^{2}}\right. \\
\left.-\left(1-r^{2}\right) \sum_{k=1}^{\infty}\left(\frac{z}{r^{2 k}} \log \left(1-\frac{r^{2(n+k)}}{z \bar{\zeta}}\right)-\frac{1}{r^{2 k} z} \log \left(1-\frac{r^{2(n+k)} z}{\zeta}\right)\right)\right] .
\end{gathered}
$$


Hence

$$
\begin{gathered}
2 \operatorname{Re}\left[\left(1-r^{2 n}\right) \zeta \frac{1}{\pi} \int_{R} \frac{1}{r^{2 n} \widetilde{\zeta}-\zeta} G_{1}(z, \tilde{\zeta}) d \tilde{\xi} d \widetilde{\eta}\right] \\
(17)=2 \frac{\left(1-r^{2 n}\right)}{r^{2 n}} \operatorname{Re}\left[\left(\frac{\bar{\zeta}}{\bar{z}}-z \bar{\zeta}\right) \log \left(1-\frac{r^{2 n} z}{\zeta}\right)+\left(1-r^{2}\right) r^{2 n} \frac{\log |z|^{2}}{\log r^{2}}\right. \\
\left.-\left(1-r^{2}\right) \sum_{k=1}^{\infty}\left(\frac{z \bar{\zeta}}{r^{2 k}} \log \left(1-\frac{r^{2(n+k)}}{z \bar{\zeta}}\right)-\frac{\zeta}{r^{2 k} z} \log \left(1-\frac{r^{2(n+k)} z}{\zeta}\right)\right)\right]
\end{gathered}
$$

Combining the results of all evaluations (formulas (13)-(17)), one obtains

$$
\begin{aligned}
& \widehat{h}_{2}(z, \zeta)=2 \operatorname{Re}\left[\frac { \operatorname { l o g } | \zeta | ^ { 2 } } { \operatorname { l o g } r ^ { 2 } } \left(1-|z|^{2}+z \bar{\zeta} \log |z|^{2}-\left(1-r^{2}\right) \frac{\log |z|^{2}}{\log r^{2}}\right.\right. \\
& \left.-\frac{r^{2}}{1-r^{2}} \log r^{2}\left(\frac{\bar{\zeta}}{\bar{z}}-z \bar{\zeta}\right)\right)+\left(1-|\zeta|^{2}\right)\left(\left(1-r^{2}\right) \frac{\log |z|^{2}}{\log r^{2}}+\left(\frac{1}{z \bar{\zeta}}-\frac{\bar{z}}{\bar{\zeta}}\right)\right. \\
& \left.\times \log (1-z \bar{\zeta})-\left(1-r^{2}\right) \sum_{k=1}^{\infty}\left(\frac{z}{r^{2 k} \zeta} \log \left(1-\frac{r^{2 k} \zeta}{z}\right)-\frac{1}{r^{2 k} z \bar{\zeta}} \log \left(1-r^{2 k} z \bar{\zeta}\right)\right)\right) \\
& -\sum_{n=1}^{\infty}\left[\frac { ( r ^ { 2 n } | \zeta | ^ { 2 } - 1 ) } { r ^ { 2 n } } \left(\left(1-r^{2}\right) r^{2 n} \frac{\log |z|^{2}}{\log |r|^{2}}+\left(\frac{1}{z \bar{\zeta}}-\frac{\bar{z}}{\bar{\zeta}}\right) \log \left(1-r^{2 n} z \bar{\zeta}\right)\right.\right. \\
& \left.-\left(1-r^{2}\right) \sum_{k=1}^{\infty}\left(\frac{z}{r^{2 k} \zeta} \log \left(1-\frac{r^{2(n+k)} \zeta}{z}\right)-\frac{1}{r^{2 k} z \bar{\zeta}} \log \left(1-r^{2(n+k)} z \bar{\zeta}\right)\right)\right) \\
& +\left(r^{2 n}-|\zeta|^{2}\right)\left(\frac{z}{\zeta} \log |z|^{2}+\frac{r^{2} \log r^{2}}{1-r^{2}}\left(\frac{\bar{z}}{\overline{\bar{\zeta}}}-\frac{1}{z \bar{\zeta}}\right)+\left(\frac{\bar{z}}{\bar{\zeta}}-\frac{r^{2}}{z \bar{\zeta}}\right) \log \left(1-\frac{r^{2 n}}{z \bar{\zeta}}\right)\right. \\
& -\left(1-r^{2}\right) \frac{z}{\zeta} \log \left(1-\frac{r^{2 n} z}{\zeta}\right)+\left(1-r^{2}\right) \sum_{k=1}^{\infty}\left(\frac{r^{2 k}}{z \bar{\zeta}} \log \left(1-\frac{r^{2(n+k)}}{z \bar{\zeta}}\right)\right. \\
& \left.\left.-\frac{r^{2 k} z}{\zeta} \log \left(1-\frac{r^{2(n+k)} z}{\zeta}\right)\right)\right)+\left(1-r^{2 n}\right)\left(z \bar{\zeta} \log |z|^{2}-\frac{r^{2} \log r^{2}}{1-r^{2}}\left(\frac{\bar{\zeta}}{\bar{z}}-z \bar{\zeta}\right)\right. \\
& -\left(1-r^{2}\right) z \bar{\zeta} \log \left(1-r^{2 n} z \bar{\zeta}\right)+\left(z \bar{\zeta}-\frac{r^{2} \bar{\zeta}}{\bar{z}}\right) \log \left(1-\frac{r^{2 n} \zeta}{z}\right)
\end{aligned}
$$




$$
\begin{gathered}
\left.+\left(1-r^{2}\right) \sum_{k=1}^{\infty}\left(\frac{r^{2 k} \zeta}{z} \log \left(1-\frac{r^{2(n+k)} \zeta}{z}\right)-r^{2 k} z \bar{\zeta} \log \left(1-r^{2(n+k)} z \bar{\zeta}\right)\right)\right) \\
-\frac{\left(1-r^{2 n}\right)}{r^{2 n}}\left(\left(1-r^{2}\right) r^{2 n} \frac{\log |z|^{2}}{\log r^{2}}+\left(\frac{\bar{\zeta}}{\bar{z}}-z \bar{\zeta}\right) \log \left(1-\frac{r^{2 n} z}{\zeta}\right)\right. \\
\left.\left.\left.-\left(1-r^{2}\right) \sum_{k=1}^{\infty}\left(\frac{z \bar{\zeta}}{r^{2 k}} \log \left(1-\frac{r^{2(n+k)}}{z \bar{\zeta}}\right)-\frac{\zeta}{r^{2 k} z} \log \left(1-\frac{r^{2(n+k)} z}{\zeta}\right)\right)\right)\right]\right] .
\end{gathered}
$$

Simplifying the expression for $\widehat{h}_{2}(z, \zeta)$, using the representation (8) and formula (7), the following main formula for the biharmonic Green function of the circular ring domain $R$

$$
\begin{gathered}
\widehat{G}_{2}(z, \zeta)=|\zeta-z|^{2} \\
\times\left[\frac{\log |z|^{2} \log |\zeta|^{2}}{\log r^{2}}-\log \left|\frac{\zeta-z}{1-z \bar{\zeta}} \prod_{k=1}^{\infty} \frac{\left(z-r^{2 k} \zeta\right)\left(\zeta-r^{2 k} z\right)}{\left(z \bar{\zeta}-r^{2 k}\right)\left(1-r^{2 k} z \bar{\zeta}\right)}\right|^{2}\right] \\
+2 \operatorname{Re}\left[\frac{\left(1-|z|^{2}\right) \log |\zeta|^{2}}{\log r^{2}}+\frac{\left(1-|\zeta|^{2}\right) \log |z|^{2}}{\log r^{2}}-\frac{r^{2}\left(1-|z|^{2}\right)}{1-r^{2}} \frac{\zeta}{z} \log |\zeta|^{2}\right. \\
-\frac{r^{2}\left(1-|\zeta|^{2}\right)}{1-r^{2}} \frac{z}{\zeta} \log |z|^{2}+\frac{z \bar{\zeta} \log |z|^{2} \log |\zeta|^{2}}{\log r^{2}}-\frac{\left(1-r^{2}\right) \log |z|^{2} \log |\zeta|^{2}}{\left(\log r^{2}\right)^{2}} \\
+\left(\frac{r^{2}}{1-r^{2}}\right)^{2} \frac{\left(1-|z|^{2}\right)\left(1-|\zeta|^{2}\right) \log r^{2}}{z \bar{\zeta}}+\left(1-|\zeta|^{2}\right)\left[\frac{1-|z|^{2}}{z \bar{\zeta}} \log (1-z \bar{\zeta})\right. \\
\left.-\left(1-r^{2}\right) \sum_{n=1}^{\infty}\left(\frac{z}{r^{2 n} \zeta} \log \left(1-\frac{r^{2 n} \zeta}{z}\right)-\frac{1}{r^{2 n} z \bar{\zeta}} \log \left(1-r^{2 n} z \bar{\zeta}\right)\right)\right] \\
(18) \quad-\sum_{n=1}^{\infty} \frac{\left(r^{2 n}|\zeta|^{2}-1\right)}{r^{2 n}} \frac{\left(1-|z|^{2}\right)}{z \bar{\zeta}} \log \left(1-r^{2 n} z \bar{\zeta}\right) \\
-\sum_{n=1}^{\infty}\left(r^{2 n}-|\zeta|^{2}\right)\left[\frac{|z|^{2}-r^{2}}{z \bar{\zeta}} \log \left(1-\frac{r^{2 n}}{z \bar{\zeta}}\right)-\left(1-r^{2}\right) \frac{z}{\zeta} \log \left(1-\frac{r^{2 n} z}{\zeta}\right)\right] \\
-\sum_{n=1}^{\infty}\left(1-r^{2 n}\right)\left[\frac{\left(|z|^{2}-r^{2}\right) \zeta}{z} \log \left(1-\frac{r^{2 n} \zeta}{z}\right)-\left(1-r^{2}\right) z \bar{\zeta} \log \left(1-r^{2 n} z \bar{\zeta}\right)\right]
\end{gathered}
$$




$$
\begin{aligned}
& -\sum_{n=1}^{\infty} \frac{\left(1-r^{2 n}\right)}{r^{2 n}} \frac{\left(1-|z|^{2}\right) \zeta}{z} \log \left(1-\frac{r^{2 n} z}{\zeta}\right)+\left(1-r^{2}\right) \sum_{n=1}^{\infty} \sum_{k=1}^{\infty} \frac{r^{2 n}|\zeta|^{2}-1}{r^{2 n}} \\
& \times\left[\frac{z}{r^{2 k} \zeta} \log \left(1-\frac{r^{2(n+k)} \zeta}{z}\right)-\frac{1}{r^{2 k} z \bar{\zeta}} \log \left(1-r^{2(n+k)} z \bar{\zeta}\right)\right] \\
& -\left(1-r^{2}\right) \sum_{n=1}^{\infty} \sum_{k=1}^{\infty}\left(r^{2 n}-|\zeta|^{2}\right)\left[\frac{r^{2 k}}{z \bar{\zeta}} \log \left(1-\frac{r^{2(n+k)}}{z \bar{\zeta}}\right)-\frac{r^{2 k} z}{\zeta} \log \left(1-\frac{r^{2(n+k)} z}{\zeta}\right)\right] \\
& -\left(1-r^{2}\right) \sum_{n=1}^{\infty} \sum_{k=1}^{\infty}\left(1-r^{2 n}\right)\left[\frac{r^{2 k} \zeta}{z} \log \left(1-\frac{r^{2(n+k)} \zeta}{z}\right)-r^{2 k} z \bar{\zeta} \log \left(1-r^{2(n+k)} z \bar{\zeta}\right)\right] \\
& \left.+\left(1-r^{2}\right) \sum_{n=1}^{\infty} \sum_{k=1}^{\infty} \frac{1-r^{2 n}}{r^{2 n}}\left[\frac{z \bar{\zeta}}{r^{2 k}} \log \left(1-\frac{r^{2(n+k)}}{z \bar{\zeta}}\right)-\frac{\zeta}{r^{2 k} z} \log \left(1-\frac{r^{2(n+k)} z}{\zeta}\right)\right]\right]
\end{aligned}
$$

is obtained.

Remark 3.1. All series in (18) are convergent because of the properties of the logarithmic function.

In the same way as it is with the harmonic Green function, the biharmonic Green function provides a representation formula for a proper class of functions. Namely, the following result holds, see e.g. [9].

Theorem 3.2. Let $D \subset \mathrm{C}$ be a regular domain. Any $w \in C^{4}(D ; \mathrm{C}) \cap$ $C^{3}(\bar{D} ; \mathrm{C})$ can be represented as

$$
\begin{aligned}
w(z)= & -\frac{1}{4 \pi} \int_{\partial D} \partial_{\nu_{\zeta}} G_{1}(z, \zeta) w(\zeta) d s_{\zeta}-\frac{1}{4 \pi} \int_{\partial D} \partial_{\nu_{\zeta}} \widehat{G}_{2}(z, \zeta) \partial_{\zeta} \partial_{\zeta} w(\zeta) d s_{\zeta} \\
& -\frac{1}{\pi} \int_{D} \widehat{G}_{2}(z, \zeta)\left(\partial_{\zeta} \partial_{\bar{\zeta}}\right)^{2} w(\zeta) d \xi d \eta
\end{aligned}
$$

with $G_{1}(z, \zeta), \widehat{G}_{2}(z, \zeta)$ being the harmonic and the biharmonic Green functions of the domain $D$, correspondingly.

This integral representation formula provides a solution of the Dirichlet problem for the bi-Poisson equation in corresponding domains, see e.g. [9].

Theorem 3.3. Let $D \subset \mathrm{C}$ be a regular domain. The solution of the Dirichlet problem to the bi-Poisson equation in $D$

$$
\left(\partial_{z} \partial_{\bar{z}}\right)^{2} w=f \text { in } D, \quad w=\gamma_{0}, \partial_{z} \partial_{\bar{z}} w=\gamma_{1} \text { on } \partial D,
$$


where $f \in L_{p}(D ; C), p>2, \gamma_{0}, \gamma_{1} \in C(\partial D ; C)$ are given, is unique and represented by

$$
\begin{aligned}
w(z)=- & \frac{1}{4 \pi} \int_{\partial D} \partial_{\nu_{\zeta}} G_{1}(z, \zeta) \gamma_{0}(\zeta) d s_{\zeta}-\frac{1}{4 \pi} \int_{\partial D} \partial_{\nu_{\zeta}} \widehat{G}_{2}(z, \zeta) \gamma_{1}(\zeta) d s_{\zeta} \\
& -\frac{1}{\pi} \int_{D} \widehat{G}_{2}(z, \zeta) f(\zeta) d \xi d \eta .
\end{aligned}
$$

\section{REFERENCES}

1. Akhiezer, N. I., Elements of the Theory of Elliptic Functions, Translations of Math. Monographs 79, Amer. Math. Soc., Providence, RI 1990.

2. Almansi, E., Sull'integrazione dell' Equazione differenziale $\triangle^{2 n}=0$, Ann. Mat. Pura Appl. (3) 2 (1899), 1-51.

3. Begehr, H., Biharmonic Green functions, Matematiche (Catania) 61 (2006), 395-405.

4. Begehr, H., Boundary value problems in complex analysis I, Bol. Asoc. Mat. Venez. 12 (2005), 65-85.

5. Begehr, H., Complex Analytic Methods for Partial Differential Equations, World Scientific, Singapore 1994.

6. Begehr, H., Hybrid Green Functions and Related Boundary Value Problems, pp. 275-278 in: Extended Abstracts of $37^{\text {th }}$ Annual Iranian Math. Conference (AIMC), Tabriz, 2006.

7. Begehr, H., The main theorem of calculus in complex analysis, Ann. European Acad. Sci. (2005), 184-210.

8. Begehr, H., Six biharmonic Dirichlet problems in complex analysis, pp. 243-252 in: Function Spaces in Complex and Clifford Analysis, Proc. Hue 2006, Natl. Univ. Publ., Hanoi 2008.

9. Begehr, H., Vaitekhovich, T., Iterated Dirichlet Problem for the Higher Order Poisson Equation, Matematiche (Catania) 63 (2008), 139-154.

10. Courant, R., Hilbert, D., Methods of Mathematical Physics I, Interscience, New York 1953.

11. Engliš, M., Peetre, J., A Green's function for the annulus, Ann. Mat. Pura Appl. (4) 171 (1996), 313-377.

12. Gaertner, E., Basic complex boundary value problems in the upper half plane, Ph.D. thesis, FU Berlin 2006: www.diss.fu-berlin.de/diss/receive/FUDISS_thesis_000000002129.

13. Haack, W., Wendland, W., Lectures on Partial and Pfaffian Differential Equations, Pergamon Press, Oxford 1972.

14. Krausz, A., Integraldarstellungen mit Greenschen Funktionen höherer Ordnung in Gebieten und Polygebieten, Ph.D. thesis, FU Berlin 2005: www.diss.fu-berlin.de/diss/receive/FUDISS_thesis_000000001659.

15. Mityushev, V. V., Rogosin, S. V., Constuctive Methods for Linear and Non-linear Boundary Value Problems for Analytic Functions: Theory and Applications, Monographs and Surveys in Pure and Appl. Math. 108, Chapman and Hall / CRC Press, Boca Raton, FL 2000.

16. Vaitekhovich, T. S., Boundary value problems to second order complex partial differential equations in a ring domain, Šiauliai Math. Seminar 2 (2007), 117-146. 
17. Vaitsiakhovich, T., Boundary value problems for complex partial differential equations in a ring domain, Ph.D. thesis, FU Berlin 2008:

www.diss.fu-berlin.de/diss/receive/FUDISS_thesis_000000003859.

\author{
MATHEMATISCHES INSTITUT \\ FU BERLIN \\ ARNIMALLEE 3 \\ D-14195 BERLIN \\ GERMANY \\ E-mail: vaiteakhovich@mail.ru
}

